\title{
A unified approach to robust estimation in finite population sampling
}

\author{
J.-F. BEAUMONT \\ Statistics Canada, Tunney's Pasture, R.H. Coats Building, 16th floor, K1A 0T6, \\ Ottawa, Canada Jean-Francois.Beaumontestatcan.gc.ca \\ D. HAZIZA \\ Département de mathématiques et de statistique, Université de Montréal, \\ Montreal, H3C 3J7, Canada David.Haziza@umontreal.ca \\ and A. RUIZ-GAZEN \\ Toulouse School of Economics, 21, allée de Brienne, 31000 Toulouse, France \\ Anne.Ruiz-Gazen@tse-fr.eu
}

\begin{abstract}
We argue that the conditional bias associated with a sample unit can be a useful measure of influence in finite population sampling. We use the conditional bias to derive robust estimators that are obtained by downweighting the most influential sample units. Under the model-based approach to inference, our proposed robust estimator is closely related to the well-known estimator of Chambers (1986). Under the design-based approach, it possesses the desirable feature of being applicable with most sampling designs used in practice. For stratified simple random sampling, it is essentially equivalent to the estimator of Kokic \& Bell (1994). The proposed robust estimator depends on a tuning constant. In this paper, we propose a method for determining the tuning constant and show that the resulting estimator is consistent. Results from a simulation study suggest that our approach improves the efficiency of standard nonrobust estimators when the population contains units that may be influential if selected in the sample.
\end{abstract}

Keywords: Conditional bias; Design-based inference; Influence function; Modelbased inference; Outlier.

\section{Introduction}

\subsection{The influence function in infinite population sampling}

The influence function (Hampel, 1974) is well known in infinite population sampling. It is often used in the context of outlier-robust estimation to study properties of estimators or derive robust estimators. To fix ideas, let $Y_{i}(i=1, \ldots, n)$ be $n$ independent random variables having the same distribution $F$ and suppose that we are interested in estimating $\theta=t(F)$. For a given fixed value $y$, the influence 
function is defined as

$$
\operatorname{IF}(y ; t, F)=\lim _{\varepsilon \rightarrow 0} \frac{t\left\{(1-\varepsilon) F+\varepsilon \delta_{y}\right\}-t(F)}{\varepsilon},
$$

where $\delta_{y}$ is the Dirac distribution at $y$. If $\theta=t(F)$ is the mean of $F$, (1) equals $\operatorname{IF}(y ; t, F)=y-\theta$, which is unbounded. Robustness is typically achieved by choosing functionals $t(F)$, such as the median of $F$, that have a bounded influence function.

Let us now consider the estimator $\hat{\theta}=t(\hat{F})$ of $\theta$, where $\hat{F}=n^{-1} \sum_{i=1}^{n} \delta_{Y_{i}}$ is the empirical distribution function. A well known approximation (e.g., Hampel et al., 1986, p. 85) that uses the influence function (1) is

$$
\hat{\theta} \simeq \theta+\frac{1}{n} \sum_{i=1}^{n} \operatorname{IF}\left(Y_{i} ; t, F\right)
$$

with $E_{F}\left\{\operatorname{IF}\left(Y_{i} ; t, F\right)\right\}=0(i=1, \ldots, n)$. This approximation may be used to estimate the variance of $\hat{\theta}$.

\subsection{The conditional bias in infinite population sampling}

Muñoz-Pichardo et al. (1995) proposed to use the conditional bias

$$
B\left(y_{i} ; \theta\right)=E_{F}\left(\hat{\theta} \mid Y_{i}=y_{i}\right)-\theta
$$

as a measure of the influence of the $i$ th observation. Schlittgen \& Schwabe (2001) introduced the asymptotic mean sensitivity curve, defined as $\lim _{n \rightarrow \infty} n B\left(y_{i} ; \theta\right)$. Using the approximation (2) with $E_{F}\left\{\operatorname{IF}\left(Y_{i} ; t, F\right)\right\}=0$, it is straightforward to approximate (3) by

$$
B\left(y_{i} ; \theta\right) \simeq \frac{1}{n} \operatorname{IF}\left(y_{i} ; t, F\right) .
$$

The conditional bias is thus approximately proportional to the influence function $\operatorname{IF}\left(y_{i} ; t, F\right)$ and so can be viewed as an influence measure for the $i$ th observation. From (2) and (4),

$$
\hat{\theta}-\theta \simeq \sum_{i=1}^{n} B\left(Y_{i} ; \theta\right) .
$$

The conditional bias can thus be viewed as the contribution of the $i$ th observation to the error $\hat{\theta}-\theta$. Robustness is achieved by curbing the influence or contribution of the largest observations on this error. 


\subsection{Design-based approach}

In finite population sampling, inference is usually made with respect to the known probability sampling design $P(S)$ that is used to select a random sample $S$ from the finite population $U$ of size $N$. This is often called the design-based approach to inference. In this approach, only the sample inclusion indicators are random; all other quantities are treated as fixed. The influence function (1) seems irrelevant in the design-based approach because there is no underlying distribution $F$ that is assumed to have generated the population values $y_{i}(i \in U)$ at least for inference purposes.

One way of defining the influence function in finite population sampling consists of replacing $F$ by $F_{N}=\sum_{i \in U} \delta_{y_{i}} / N$ so that the finite population parameter $\theta$ can be expressed as $\theta=t\left(F_{N}\right)$. Unfortunately, this leads to an influence function that fails to account for the sampling design. For instance, if $\theta=t\left(F_{N}\right)$ is the population mean of $F_{N}$, i.e. $\theta=\sum_{i \in U} y_{i} / N$, the influence function is again $\operatorname{IF}\left(y ; t, F_{N}\right)=y-\theta$. The quantity $\operatorname{IF}\left(y_{i} ; t, F_{N}\right)=y_{i}-\theta$ is not a good measure of the influence of unit $i$ because it ignores the sampling design. As an example, suppose that unit $i$ is selected with certainty in the sample. It would seem intuitively appealing to consider an influence measure that is equal to zero for this unit. This is not the case for $\operatorname{IF}\left(y_{i} ; t, F_{N}\right)=y_{i}-\theta$.

Approximations analogous to (2) have also been developed within the designbased approach (e.g., Campbell, 1980; Gwet \& Rivest, 1992; Deville, 1999; Demnati \& Rao, 2004). While these methods are quite useful for variance estimation, they lead to influence measures that do not account for the sampling design.

Assuming with-replacement sampling, and thus independent and identically distributed observations, Zaslavsky et al. (2001) extended the definition (1) to finite population sampling under the design-based approach. Hulliger (1995) defined a sensitivity curve for probability proportional to size sampling. He took the sampling design into account by expressing the finite population parameter $\theta$ as a function of the population values of the size variable. Although the sampling design is involved in both methods, they again yield a non-zero influence measure for a unit $i$ selected with certainty in the sample. Also, it does not seem easy to generalize these methods to other sampling designs with possibly large sampling fractions.

The conditional bias might be a useful measure of influence since it can be easily extended to any inferential framework. In the next sections, we use the conditional bias to derive robust estimators in finite population sampling by downweighting the most influential sample units. We first consider the model-based approach to inference in section 2 . We then consider the design-based approach to inference in sections 3-5. 


\section{Robust best linear unbiased predictor}

In the model-based approach to inference in finite population sampling (e.g., Valliant et al., 2000), the $y$-values of the $N$ population units are assumed to be generated by some model. We denote by $X$, the known $N$-row matrix containing the vector of explanatory variables $x_{i}^{\mathrm{T}}$ in its $i$ th row. Often, a linear model is considered, for which $\left(Y_{i}-x_{i}^{\mathrm{T}} \beta\right) / \sigma_{i}(i \in U)$ given $X$ are mutually independent and have all the same distribution $F$, where $\beta$ is a vector of unknown model parameters and $\sigma_{i}$ is usually assumed to be known up to a constant factor. Furthermore, the distribution $F$ has a mean of zero and a variance of one.

A non-informative sample $s$ is selected from the finite population $U$ and is treated as fixed when making inferences. The interest is in the prediction of a function of the population $Y$-variables through the sample $Y$-variables. To fix ideas, we will assume that we are interested in predicting the random population total $\theta=\sum_{i \in U} Y_{i}$. Royall (1976) proposed the best linear unbiased predictor of $\theta$, which can be expressed as

$$
\hat{\theta}=\sum_{i \in s} w_{i} Y_{i}
$$

with the weights

$$
w_{i}=1+\frac{x_{i}^{\mathrm{T}}}{\sigma_{i}^{2}}\left(\sum_{i \in s} \frac{x_{i} x_{i}^{\mathrm{T}}}{\sigma_{i}^{2}}\right)^{-1}\left(\sum_{i \in U-s} x_{i}\right) .
$$

In this context, the conditional bias attached to unit $i$ is

$$
B_{i}\left(y_{i} ; \beta\right)=E_{F}\left(\hat{\theta}-\theta \mid s, Y_{i}=y_{i}\right) .
$$

Definition (7) is slightly different from definition (3) to account for the fact that $\theta$ is a random variable. Using [5] and noting from (6) that $\sum_{i \in s} w_{i} x_{i}=\sum_{i \in U} x_{i}$, the conditional bias (7) can be expressed as

$$
B_{i}\left(y_{i} ; \beta\right)=\left\{\begin{array}{l}
\left(w_{i}-1\right)\left(y_{i}-x_{i}^{\mathrm{T}} \beta\right) \quad(i \in s), \\
-\left(y_{i}-x_{i}^{\mathrm{T}} \beta\right) \quad(i \in U-s) .
\end{array}\right.
$$

This expression highlights that the conditional bias takes a different form depending on whether unit $i$ has been selected in the sample or not. The prediction error of (5) can be written as

$$
\hat{\theta}-\theta=\sum_{i \in U} B_{i}\left(Y_{i} ; \beta\right)
$$

Therefore, the conditional bias $B_{i}\left(Y_{i} ; \beta\right)$ can be interpreted as the contribution of unit $i$ to the prediction error of (5). Although they did not consider the conditional 
bias, Beaumont \& Rivest (2009) showed that this decomposition of the prediction error holds for any weighted estimator that satisfies the calibration equation $\sum_{i \in s} w_{i} x_{i}=\sum_{i \in U} x_{i}$. Also, the prediction variance of $\hat{\theta}$ can be expressed as

$\operatorname{var}_{F}(\hat{\theta}-\theta \mid s)=\sum_{i \in s}\left(w_{i}-1\right)^{2} \sigma_{i}^{2}+\sum_{i \in U-s} \sigma_{i}^{2}=E_{F}\left\{\sum_{i \in U} B_{i}^{2}\left(Y_{i} ; \boldsymbol{\beta}\right) \mid s\right\}$.

To construct a robust version of the best linear unbiased predictor, we first express it as:

$$
\hat{\theta}=\left\{\hat{\theta}-\sum_{i \in U} B_{i}\left(Y_{i} ; \beta\right)\right\}+\sum_{i \in U} B_{i}\left(Y_{i} ; \beta\right) .
$$

From (9), the first term on the right-hand side of (10) is equal to $\theta$ and is thus unaffected by influential units. The error comes entirely from the second term. To obtain robustness, it would be desirable to reduce the contribution of the largest $B_{i}\left(Y_{i} ; \beta\right)$ on this second term. Curbing the influence of nonsample units is not possible as their $y$-value is unknown, so their conditional bias cannot be estimated. Thus, nothing can be done at the estimation stage to protect against influential units in the nonsample portion of the population. Protection can be achieved against the occurrence of influential sample units by downweighting their contribution in the second term on the right-hand side of $(10)$. Using (8), this leads to the robust estimator:

$$
\begin{aligned}
\hat{\theta}^{\mathrm{R}}(\beta) & =\left\{\hat{\theta}-\sum_{i \in U} B_{i}\left(Y_{i} ; \beta\right)\right\}+\sum_{i \in s} \psi\left\{B_{i}\left(Y_{i} ; \beta\right)\right\}+\sum_{i \in U-s} B_{i}\left(Y_{i} ; \beta\right) \\
& =\sum_{i \in s} Y_{i}+\sum_{i \in U-s} x_{i}^{\mathrm{T}} \beta+\sum_{i \in s} \psi\left\{\left(w_{i}-1\right)\left(Y_{i}-x_{i}^{\mathrm{T}} \beta\right)\right\}
\end{aligned}
$$

where $\psi$ is any bounded function such that $\psi(z) \simeq z$ when $z$ is close to 0 . A typical choice is the Huber function, $\psi(z ; c)=\operatorname{sign}(z) \times \min (|z|, c)$, where $c$ is a positive tuning constant and $\operatorname{sign}(z)=1$, for $z \geq 0$, while $\operatorname{sign}(z)=-1$, otherwise. In general, the vector $\beta$ is unknown and must be replaced by some estimator $\hat{\beta}$, which yields the robust estimator $\hat{\theta}^{\mathrm{R}}(\hat{\beta})$. The estimator $\hat{\beta}$ could be any robust estimator developed for infinite populations, as in Chambers (1986), or could be obtained using an independent source of data, as in Kokic \& Bell (1994). In Section 5, we have empirical evidence in the design-based context that the use of a nonrobust estimator of $\beta$ leads to properties similar to those of robust estimators. We expect similar behaviour in the model-based approach. If the underlying tuning constant $c$ in the Huber $\psi$ function is large, the estimator $\hat{\theta}^{\mathrm{R}}(\hat{\beta})$ reduces to the best linear unbiased predictor for every choice of $\hat{\beta}$. 
The robust estimator $\hat{\theta}^{\mathrm{R}}(\hat{\beta})$, obtained from $(11)$, is closely related to the estimator developed by Chambers (1986) using arguments not involving the conditional bias. Chambers' estimator is:

$$
\hat{\theta}^{\mathrm{C}}(\hat{\beta})=\sum_{i \in s} Y_{i}+\sum_{i \in U-s} x_{i}^{\mathrm{T}} \hat{\beta}+\sum_{i \in s}\left(w_{i}-1\right) \hat{\sigma}_{i} \psi\left\{\left(Y_{i}-x_{i}^{\mathrm{T}} \hat{\beta}\right) / \hat{\sigma}_{i}\right\},
$$

where $\hat{\sigma}_{i}$ is a robust estimator of $\sigma_{i}$. The robust estimator given by (12) reduces the impact of large standardized residuals $\left(Y_{i}-x_{i}^{\mathrm{T}} \hat{\beta}\right) / \hat{\sigma}_{i}$ but, unlike $\theta^{\mathrm{R}}(\hat{\beta})$, does not address the combined influence of weights $\left(w_{i}-1\right) \hat{\sigma}_{i}$ and standardized residuals. Both $\hat{\theta}^{\mathrm{R}}(\hat{\beta})$ and $\hat{\theta}^{\mathrm{C}}(\hat{\beta})$ are special cases of a slightly more general estimator given in equation (6) of Beaumont \& Rivest (2009). In a design-based empirical study, they have shown a slight superiority of $\hat{\theta}^{\mathrm{R}}(\hat{\beta})$ over $\hat{\theta}^{\mathrm{C}}(\hat{\beta})$.

\section{Robust Horvitz-Thompson estimator}

\subsection{General framework}

We denote by $I_{i}(i \in U)$ the $N$ sample inclusion indicators such that $I_{i}=1$, if $i \in S$, and $I_{i}=0$, otherwise. Let us suppose again that we are interested in estimating the finite population total $\theta=\sum_{i \in U} y_{i}$ and that we consider the Horvitz-Thompson estimator $\hat{\theta}^{\mathrm{HT}}=\sum_{i \in S} d_{i} y_{i}$, where $d_{i}=1 / \pi_{i}$ is the design weight attached to unit $i$ and $\pi_{i}=\operatorname{pr}\left(I_{i}=1\right)$ is its first-order inclusion probability. The Horvitz-Thompson estimator is design-unbiased; i.e., $E_{P}\left(\hat{\theta}^{\mathrm{HT}}\right)=\theta$, where the subscript $P$ indicates that the expectation is evaluated with respect to the sampling design. For a sample unit, the conditional bias of the Horvitz-Thompson estimator is defined as

$$
B_{1 i}^{\mathrm{HT}}=E_{P}\left(\hat{\theta}^{\mathrm{HT}} \mid I_{i}=1\right)-\theta=\sum_{j \in U}\left(\frac{\pi_{i j}}{\pi_{i} \pi_{j}}-1\right) y_{j},
$$

where $\pi_{i j}=\operatorname{pr}\left(I_{i}=1, I_{j}=1\right)$ denotes the second-order inclusion probability of units $i$ and $j$. Using $E_{P}\left(\hat{\theta}^{\mathrm{HT}}\right)=\theta$, it is not difficult to show that the conditional bias for a nonsample unit is

$$
B_{0 i}^{\mathrm{HT}}=E_{P}\left(\hat{\theta}^{\mathrm{HT}} \mid I_{i}=0\right)-\theta=-\left(d_{i}-1\right)^{-1} B_{1 i}^{\mathrm{HT}} .
$$

See also Moreno-Rebollo et al. (1999) and Moreno-Rebollo et al. (2002). The conditional bias $B_{1 i}^{\mathrm{HT}}$ is equal to zero when $\pi_{i}=1$. This is an intuitively appealing property. The design variance of the Horvitz-Thompson estimator can be expressed as

$$
\operatorname{var}_{P}\left(\hat{\theta}^{\mathrm{HT}}\right)=E_{P}\left(\hat{\theta}^{\mathrm{HT}}-\theta\right)^{2}=\sum_{i \in U} B_{1 i}^{\mathrm{HT}} y_{i} .
$$


Therefore, the design variance of $\hat{\theta}^{\mathrm{HT}}$ is identically equal to zero if and only if $B_{1 i}^{\mathrm{HT}}=0$ for all $i \in U$. For instance, this occurs when the sample size is fixed and $y_{i} / \pi_{i}=a$ for some constant $a$. This also occurs when a census is conducted.

For any sampling design, we can write

$$
\hat{\theta}^{\mathrm{HT}}-\theta=\sum_{i \in S} B_{1 i}^{\mathrm{HT}}+\sum_{i \in U-S} B_{0 i}^{\mathrm{HT}}+\left(\sum_{i \in S} d_{i} A_{i}-\sum_{i \in U} A_{i}\right),
$$

where

$$
A_{i}=-\left(1-\pi_{i}\right)^{-1} \sum_{\substack{j \in U \\ j \neq i}}\left(\frac{\pi_{i j}-\pi_{i} \pi_{j}}{\pi_{j}}\right) y_{j}
$$

Provided the term in parentheses on the right hand side of (14) is equal to zero, the conditional bias can be interpreted as the contribution of unit $i$ to the sampling error of the Horvitz-Thompson estimator. This is satisfied for Poisson sampling. For stratified simple random sampling and fixed-size high-entropy sampling with varying first-order inclusion probabilities, it can be shown that the term $\hat{\theta}^{\mathrm{HT}}-\theta$ is $O_{p}\left(N n^{-1 / 2}\right)$, whereas the term in parentheses on the right hand side of 114 is $O_{p}\left(n^{-1 / 2}\right)$. Thus, in these two cases, we can write

$$
\hat{\theta}^{\mathrm{HT}}-\theta \simeq \sum_{i \in S} B_{1 i}^{\mathrm{HT}}+\sum_{i \in U-S} B_{0 i}^{\mathrm{HT}}
$$

Assuming a sampling design for which $(15)$ holds and using an argument similar to the one that led to $[11$, we obtain the following robust alternative to the HorvitzThompson estimator:

$$
\hat{\theta}^{\mathrm{RHT}}=\hat{\theta}^{\mathrm{HT}}-\sum_{i \in S} B_{1 i}^{\mathrm{HT}}+\sum_{i \in S} \psi\left(B_{1 i}^{\mathrm{HT}}\right) .
$$

The conditional bias $B_{1 i}^{\mathrm{HT}}$ in 16 depends in general on unknown population parameters that should be estimated either using current data or an independent source of data. The resulting estimated conditional bias is denoted by $\hat{B}_{1 i}^{\mathrm{HT}}$ and replaces $B_{1 i}^{\mathrm{HT}}$ in 16 when it is unknown. Estimation of $B_{1 i}^{\mathrm{HT}}$ using current data is discussed in Section 3.3.

\subsection{Examples}

For Poisson sampling, $\pi_{i j}=\pi_{i} \pi_{j}, i \neq j$ and the conditional bias in (13) reduces to

$$
B_{1 i}^{\mathrm{HT}}=\left(d_{i}-1\right) y_{i}
$$


Unit $i$ has a large influence if its design weight, $d_{i}$, is large and/or if its $y$-value, $y_{i}$, is large. The conditional bias is known for all the sample units and so it does not need to be estimated.

For without-replacement stratified simple random sampling, it is straightforward to show that

$$
B_{1 i}^{\mathrm{HT}}=\frac{N_{h}}{N_{h}-1}\left(\frac{N_{h}}{n_{h}}-1\right)\left(y_{i}-\bar{Y}_{h}\right), \quad \text { for } i \in U_{h}
$$

where $n_{h}$ is the sample size in stratum $h$ and $\bar{Y}_{h}=N_{h}^{-1} \sum_{i \in U_{h}} y_{i}$ with $U_{h}$ denoting the set of population units in stratum $h$ and $N_{h}$ the size of $U_{h}$. From (17), an observation in stratum $h$ has a large influence when it is far from the stratum mean, $\bar{Y}_{h}$. Ignoring the factor $N_{h} /\left(N_{h}-1\right)$ in 17$)$ and considering the one-sided Huber function $\psi(z ; c)=\min (z, c)$, the resulting robust estimator 16 becomes equivalent to the Winsorized estimator of Kokic \& Bell (1994).

For high entropy fixed-size sampling designs, Hájek (1981) proposed the following approximation to $\pi_{i j}$ :

$$
\pi_{i j} \simeq \pi_{i} \pi_{j}\left\{1-D^{-1}\left(1-\pi_{i}\right)\left(1-\pi_{j}\right)\right\},
$$

where $D=\sum_{l \in U} \pi_{l}\left(1-\pi_{l}\right)$. This approximation is more accurate for large sample and population sizes. Using (18) in (13), we can approximate the conditional bias attached to unit $i$ by

$$
B_{1 i}^{\mathrm{HT}} \simeq\left(d_{i}-1\right)\left[\left\{1+D^{-1} \pi_{i}\left(1-\pi_{i}\right)\right\} y_{i}-\phi \pi_{i}\right],
$$

where $\phi=D^{-1} \sum_{j \in U}\left(1-\pi_{j}\right) y_{j}$. Under mild regularity conditions, the term $D^{-1} \pi_{i}\left(1-\pi_{i}\right)$ in $\sqrt{19}$ is $O\left(N^{-1}\right)$. Assuming that the population size $N$ is large, we can neglect this term from (19), which leads to

$$
B_{1 i}^{\mathrm{HT}} \simeq\left(d_{i}-1\right)\left(y_{i}-\phi \pi_{i}\right)
$$

From (20), it is clear that unit $i$ has a large influence if its weight, $d_{i}$, is large or if its residual, $\left(y_{i}-\phi \pi_{i}\right)$, is large.

\subsection{Estimation of the conditional bias}

In general, the conditional bias, $B_{1 i}^{\mathrm{HT}}$, must be estimated. One possibility consists of using the conditionally design-unbiased estimator of $B_{1 i}^{\mathrm{HT}}$ :

$$
\hat{B}_{1 i}^{\mathrm{HT}}=\sum_{j \in S}\left(\frac{\pi_{i j}-\pi_{i} \pi_{j}}{\pi_{j} \pi_{i j}}\right) y_{j},
$$


provided $\pi_{i j}>0$ for all $j \in U$. That is, $E_{P}\left(\hat{B}_{1 i}^{\mathrm{HT}} \mid I_{i}=1\right)=B_{1 i}^{\mathrm{HT}}$. In the special case of stratified simple random sampling, the estimated conditional bias (21) reduces to

$$
\hat{B}_{1 i}^{\mathrm{HT}}=\frac{n_{h}}{n_{h}-1}\left(\frac{N_{h}}{n_{h}}-1\right)\left(y_{i}-\bar{y}_{h}\right) \quad \text { for } i \in U_{h},
$$

where $\bar{y}_{h}=n_{h}^{-1} \sum_{i \in S_{h}} y_{i}$ denotes the sample mean in stratum $h$ and $S_{h}$ is the set of sample units in stratum $h$. It is clear that 22) was obtained from (17) by replacing $N_{h} /\left(N_{h}-1\right)$ with $n_{h} /\left(n_{h}-1\right)$ and the true stratum mean $\bar{Y}_{h}$ with the sample mean $\bar{y}_{h}$.

Alternatively, the stratum mean $\bar{Y}_{h}$ in $(17)$ could be replaced by a robust estimator, the median, say, which leads to

$$
\hat{B}_{1 i}^{\mathrm{HT}}=\frac{N_{h}}{N_{h}-1}\left(\frac{N_{h}}{n_{h}}-1\right)\left(y_{i}-m_{h}\right) \quad \text { for } i \in U_{h},
$$

where $m_{h}$ is the sample median in stratum $h$. Similarly, in the case of a high entropy sampling design, the coefficient $\phi$ in 20) can be estimated by a standard nonrobust estimator or by a robust estimator such as an $M$-estimator. In our empirical study in Section 5, it turns out that the choice of an estimator of the conditional bias makes little difference in terms of relative bias and relative efficiency, at least in our scenarios.

\subsection{Choice of the tuning constant}

The $\psi$-function in (16) usually depends on a tuning constant $c$. A suitable value for $c$ is sometimes determined by minimizing an estimator of the mean square error of the robust estimator (e.g., Hulliger, 1995; Kokic \& Bell, 1994; and Beaumont $\&$ Rivest, 2009). We propose an alternative approach that consists of finding the value of $c$ that minimizes

$$
\max \left\{\left|\hat{B}_{1 i}^{\mathrm{RHT}}(c)\right| ; i \in S\right\},
$$

where $\hat{B}_{1 i}^{\mathrm{RHT}}(c)$ is an estimator of the conditional bias of the robust HorvitzThompson estimator. From (16), this conditional bias can be written as

$$
\begin{aligned}
B_{1 i}^{\mathrm{RHT}}(c) & =E_{P}\left(\hat{\theta}^{\mathrm{RHT}}(c) \mid I_{i}=1\right)-\theta \\
& =B_{1 i}^{\mathrm{HT}}+E_{P}\left[\sum_{j \in S}\left\{\psi\left(\hat{B}_{1 j}^{\mathrm{HT}} ; c\right)-\hat{B}_{1 j}^{\mathrm{HT}}\right\} \mid I_{i}=1\right] .
\end{aligned}
$$


A conditionally unbiased estimator of the last conditional expectation in 25 is simply $\Delta(c)=\sum_{i \in S}\left\{\psi\left(\hat{B}_{1 i}^{\mathrm{HT}} ; c\right)-\hat{B}_{1 i}^{\mathrm{HT}}\right\}$. This yields the estimator

$$
\hat{B}_{1 i}^{\mathrm{RHT}}(c)=\hat{B}_{1 i}^{\mathrm{HT}}+\Delta(c) .
$$

Let $\hat{B}_{\min }^{\mathrm{HT}}=\min \left(\hat{B}_{1 i}^{\mathrm{HT}} ; i \in S\right)$ and $\hat{B}_{\max }^{\mathrm{HT}}=\max \left(\hat{B}_{1 i}^{\mathrm{HT}} ; i \in S\right)$. Because $\Delta(c)$ does not depend on $i$, the value of $c$ that minimizes (24), denoted by $c_{\min }$, must be such that

$$
-\left\{\hat{B}_{\min }^{\mathrm{HT}}+\Delta\left(c_{\min }\right)\right\}=\hat{B}_{\max }^{\mathrm{HT}}+\Delta\left(c_{\min }\right),
$$

for any choice of the function $\psi$. As a result, $\Delta\left(c_{\min }\right)=-\left(\hat{B}_{\min }^{\mathrm{HT}}+\hat{B}_{\max }^{\mathrm{HT}}\right) / 2$. The robust estimator 16 ) can be expressed as $\hat{\theta}^{\mathrm{RHT}}(c)=\hat{\theta}^{\mathrm{HT}}+\Delta(c)$, for any value of $c$. Therefore, our proposed robust estimator reduces to

$$
\hat{\theta}^{\mathrm{RHT}}\left(c_{\min }\right)=\hat{\theta}^{\mathrm{HT}}+\Delta\left(c_{\min }\right)=\hat{\theta}^{\mathrm{HT}}-\frac{1}{2}\left(\hat{B}_{\min }^{\mathrm{HT}}+\hat{B}_{\max }^{\mathrm{HT}}\right) .
$$

The robust estimator (26) can be obtained without actually computing the value $c_{\text {min }}$ so that no iterative process is required. In contrast, determining the value of $c$ by minimizing an estimator of the mean square error is much more computerintensive and is often achieved by using simplifying assumptions.

Remark: The robust estimator $\hat{\theta}^{\mathrm{RHT}}\left(c_{\min }\right)$ in 26 can be justified without requiring the decomposition of the sampling error (15) to hold approximately. To observe this, let us consider a class of estimators of the form $\hat{\theta}^{\mathrm{RHT}}\left(\lambda_{S}\right)=$ $\hat{\theta}^{\mathrm{HT}}+\lambda_{S}$ for which estimator 16 is a special case. Similarly as above, an estimator of the conditional bias $B_{1 i}^{\mathrm{RHT}}\left(\lambda_{S}\right)=E_{P}\left\{\hat{\theta}^{\mathrm{RHT}}\left(\lambda_{S}\right)-\theta \mid I_{i}=1\right\}$ is: $\hat{B}_{1 i}^{\mathrm{HT}}\left(\lambda_{S}\right)=\hat{B}_{1 i}^{\mathrm{HT}}+\lambda_{S}$. The statistic $\lambda_{S}$ can be determined by minimizing $\max \left(\left|\hat{B}_{1 i}^{\mathrm{HT}}+\lambda_{S}\right| ; i \in S\right)$. Let $\lambda_{S, \text { min }}$ denote the solution to this minimization problem. The resulting robust estimator, $\hat{\theta}^{\mathrm{RHT}}\left(\lambda_{S, \mathrm{~min}}\right)$, is equal to the right side of (26).

Remark: Even though computing $c_{\min }$ is not necessary to obtain the right side of (26), it may still be useful to compute it for mean square error estimation (see Section 3.5) or for the implementation of the robust estimator (see Section 6). The value $c_{\min }$ is the solution in $c$ to the equation $\Delta(c)=-\left(\hat{B}_{\min }^{\mathrm{HT}}+\hat{B}_{\max }^{\mathrm{HT}}\right) / 2$. It can be shown that, if the Huber $\psi$ function is used, there always exists a solution to this equation although it is not necessarily unique. If this occurs, one can choose the largest value of $c$ that solves the equation. The proof is given in the appendix.

Let us now assume the standard conditions: (i) $\hat{\theta}^{\mathrm{HT}}-\theta=O_{p}\left(\mathrm{Nn}^{-1 / 2}\right)$; and (ii) $\max \left(\left|\hat{B}_{1 i}^{\mathrm{HT}}\right| ; i \in S\right)=O_{p}\left(N n^{-1}\right)$. The first condition simply requires 
that the Horvitz-Thompson estimator is design-consistent and the second condition should normally be satisfied provided that $d_{i}=O\left(N n^{-1}\right)$ and $d_{i} d_{j}\left(\pi_{i j}-\pi_{i} \pi_{j}\right)=$ $O\left(n^{-1}\right)$ if $i \neq j$. This is true when the estimator 21) is used. Under these conditions, our robust estimator $\hat{\theta}^{\mathrm{RHT}}\left(c_{\mathrm{min}}\right)$ is design-consistent. This can be seen by considering the decomposition

$$
\hat{\theta}^{\mathrm{RHT}}\left(c_{\min }\right)-\theta=\left(\hat{\theta}^{\mathrm{HT}}-\theta\right)-\frac{1}{2}\left(\hat{B}_{\min }^{\mathrm{HT}}+\hat{B}_{\max }^{\mathrm{HT}}\right),
$$

and by noting that the second term on the right side of the previous equation is $O_{p}\left(N n^{-1}\right)$, which is asymptotically negligible compared with the first term so that $\hat{\theta}^{\mathrm{RHT}}\left(c_{\min }\right)-\theta=O_{p}\left(N n^{-1 / 2}\right)$. Note that 26 is not only consistent but also asymptotically equivalent to the Horvitz-Thompson estimator.

\subsection{Mean square error estimation}

The estimation of the mean square error of robust estimators is necessary in practice to have an indication of whether they are more efficient than non-robust estimators. The design mean square error of $\hat{\theta}^{\mathrm{RHT}}$ can be written as

$\operatorname{MSE}_{P}\left(\hat{\theta}^{\mathrm{RHT}}\right)=\operatorname{var}_{P}\left(\hat{\theta}^{\mathrm{RHT}}\right)+\left\{\mathrm{E}_{P}\left(\hat{\theta}^{\mathrm{RHT}}-\hat{\theta}^{\mathrm{HT}}\right)^{2}-\operatorname{var}_{P}\left(\hat{\theta}^{\mathrm{RHT}}-\hat{\theta}^{\mathrm{HT}}\right)\right\}$.

The two terms within the brackets in 27) represent an approximation of the square design bias of $\hat{\theta}^{\mathrm{RHT}}$. Similarly to Gwet \& Rivest (1992), we suggest the mean square error estimator

$\operatorname{mse}_{P}\left(\hat{\theta}^{\mathrm{RHT}}\right)=v_{P}\left(\hat{\theta}^{\mathrm{RHT}}\right)+\max \left\{0,\left(\hat{\theta}^{\mathrm{RHT}}-\hat{\theta}^{\mathrm{HT}}\right)^{2}-v_{P}\left(\hat{\theta}^{\mathrm{RHT}}-\hat{\theta}^{\mathrm{HT}}\right)\right\}$,

where $v_{P}\left(\hat{\theta}^{\mathrm{RHT}}\right)$ and $v_{P}\left(\hat{\theta}^{\mathrm{RHT}}-\hat{\theta}^{\mathrm{HT}}\right)$ are design-consistent estimators of $\operatorname{var}_{P}\left(\hat{\theta}^{\mathrm{RHT}}\right)$ and $\operatorname{var}_{P}\left(\hat{\theta}^{\mathrm{RHT}}-\hat{\theta}^{\mathrm{HT}}\right)$, respectively. Replication variance estimation techniques, such as the bootstrap, may provide a practical way of obtaining $v_{P}\left(\hat{\theta}^{\mathrm{RHT}}\right)$ and $v_{P}\left(\hat{\theta}^{\mathrm{RHT}}-\hat{\theta}^{\mathrm{HT}}\right)$. Assuming $c$ is fixed, there are a number of methods that can be used and that are typically implemented by generating bootstrap weights; e.g., Rao et al. (1992) and Beaumont \& Patak (2012).

If the tuning constant $c$ is determined by finding the value that minimizes (24), the robust Horvitz-Thompson estimator reduces to (26). It is not obvious how to properly bootstrap $\hat{B}_{\min }^{\mathrm{HT}}$ and $\hat{B}_{\max }^{\mathrm{HT}}$ in 26 . Indeed, we do not know of any method that could be used to estimate the design variance of $\hat{\theta}^{\mathrm{RHT}}\left(c_{\min }\right)$ because of the max function in 24). Further research is needed to obtain a proper variance estimator for $\hat{\theta}^{\mathrm{RHT}}\left(c_{\mathrm{min}}\right)$. For now, we suggest treating $c_{\text {min }}$ as being fixed, which 
is often done in the literature. This implies that the value $c_{\min }$ must actually be computed even though it is not necessary for the computation of (26).

The tuning constant $c$ may also be determined by minimizing (28). If the bootstrap is used to obtain the mean square error estimate, a double bootstrap may be required to account for the variability of $\hat{\theta}^{\mathrm{RHT}}$ that is due to estimating $c$. It is not clear how to properly perform double bootstrapping in survey sampling. Further investigations are needed on this topic. To simplify the estimation of the mean square error, we again suggest treating the estimated tuning constant as fixed.

\section{Robust generalized regression estimator}

In this section, we consider the case of the generalized regression estimator of

$\theta=\sum_{i \in U} y_{i}$. We assume that a vector of auxiliary variables $x_{i}$ is available for all $i \in S$ and that the population total of the $x$-vector, $\sum_{i \in U} x_{i}$, is known. The generalized regression estimator of $\theta$ is

$$
\hat{\theta}^{\mathrm{G}}=\sum_{i \in S} d_{i} y_{i}+\left(\sum_{i \in U} x_{i}-\sum_{i \in S} d_{i} x_{i}\right)^{\mathrm{T}} \hat{\gamma}
$$

where

$$
\hat{\gamma}=\left(\sum_{j \in S} d_{j} \nu_{j}^{-1} x_{j} x_{j}^{\mathrm{T}}\right)^{-1} \sum_{j \in S} d_{j} \nu_{j}^{-1} x_{j} y_{j}
$$

and $\nu_{i}$ is a known constant attached to unit $i$. Using a first-order Taylor expansion and neglecting the higher order terms, we can write

$$
\hat{\theta}^{\mathrm{G}}-\theta \simeq \sum_{i \in S} d_{i} E_{i}-\sum_{i \in U} E_{i}
$$

where $E_{i}=y_{i}-x_{i}^{\mathrm{T}} \gamma$ denotes the census residual attached to unit $i$ with

$$
\gamma=\left(\sum_{j \in U} \nu_{j}^{-1} x_{j} x_{j}^{\mathrm{T}}\right)^{-1} \sum_{j \in U} \nu_{j}^{-1} x_{j} y_{j} .
$$

It follows from (31) that the asymptotic conditional bias attached to unit $i$ of the generalized regression estimator is

$$
B_{1 i}^{\mathrm{G}}=E_{P}\left(\hat{\theta}^{\mathrm{G}} \mid I_{i}=1\right)-\theta \simeq \sum_{j \in U}\left(\frac{\pi_{i j}}{\pi_{i} \pi_{j}}-1\right) E_{j} .
$$


The conditional bias, $B_{1 i}^{\mathrm{G}}$, with respect to the generalized regression estimator is identical to $(13)$ with $y_{i}$ replaced by $E_{i}$. Using an argument similar to the one that led to 11 or 16 , we obtain a robust version of $\hat{\theta}^{\mathrm{G}}$ as

$$
\hat{\theta}^{\mathrm{RG}}=\hat{\theta}^{\mathrm{G}}-\sum_{i \in S} B_{1 i}^{\mathrm{G}}+\sum_{i \in S} \psi\left(B_{1 i}^{\mathrm{G}}\right) .
$$

Once again, the conditional bias $B_{1 i}^{\mathrm{G}}$ in 33 depends in general on unknown population parameters that should be estimated using current data or an independent source of data. In the former case, one needs to estimate $\gamma$. One could use the weighted least square estimator $\hat{\gamma}$ given by 30 or a robust estimator such as an $M$-estimator. The resulting estimated conditional bias is denoted by $\hat{B}_{1 i}^{\mathrm{G}}$ and replaces $B_{1 i}^{\mathrm{G}}$ in 33 .

Similarly to Section 3.4 , the choice of the tuning constant can be obtained by minimizing the maximum estimated conditional bias of the robust generalized regression estimator. The resulting robust estimator is

$$
\hat{\theta}^{\mathrm{RG}}\left(c_{\min }\right)=\hat{\theta}^{\mathrm{G}}-\frac{1}{2}\left(\hat{B}_{\min }^{\mathrm{G}}+\hat{B}_{\max }^{\mathrm{G}}\right),
$$

where $\hat{B}_{\min }^{\mathrm{G}}=\min \left(\hat{B}_{1 i}^{\mathrm{G}} ; i \in S\right)$ and $\hat{B}_{\max }^{\mathrm{G}}=\max \left(\hat{B}_{1 i}^{\mathrm{G}} ; i \in S\right)$. The robust estimator (33) has a form similar to (16). As a result, estimation of the mean square error can be done in a similar fashion as in Section 3.5. The design consistency of $\hat{\theta}^{\mathrm{RG}}\left(c_{\min }\right)$ follows under regularity conditions similar to those needed for establishing the consistency of $\hat{\theta}^{\mathrm{RHT}}\left(c_{\min }\right)$.

\section{Empirical study}

We conducted a limited simulation to investigate the performance of the proposed robust estimator in terms of relative bias and relative efficiency. We generated six populations, each consisting of an auxiliary variable $x$ and a variable of interest $y$. In each population, the $x$-values were first generated from a Gamma distribution with mean 50 and variance 500.

In the first three populations, of size 500, 1000 and 5000, respectively, the $y$ values were generated according to the ratio model, $y_{i}=2 x_{i}+3.7 x_{i}^{1 / 2} \varepsilon_{i}$, where the error terms $\varepsilon_{i}$ were generated from a standard normal distribution, which lead to a coefficient of determination approximately equal to 0.75 . These populations did not contain any outlier.

In the last three populations, also of size 500, 1000 and 5000, respectively, the $y$-values were generated according to the mixture model, $y_{i}=\tau_{i}\left(2 x_{i}+3.7 x_{i}^{1 / 2} \varepsilon_{i}\right)+$ 
$\left(1-\tau_{i}\right) z_{i}$, where the $z$-values were independently generated from a normal distribution with mean 1200 and standard deviation 200 and the $\tau_{i}$ 's were independently generated from a Bernoulli distribution with probability $p=0.98$. That is, these populations contained approximately $2 \%$ of outliers.

From each population, we selected $R=10,000$ samples according to Poisson sampling with inclusion probabilities, $\pi_{i}$, proportional to $x_{i}$; that is, $\pi_{i}=$ $\tilde{n} x_{i} / \sum_{i \in U} x_{i}$, where $\tilde{n}$ denotes the expected sample size. The expected sampling fractions, $f=\tilde{n} / N$, were set to 0.02 and 0.10 .

The choice of Poisson sampling in our simulation study is justified by the fact that it makes sample coordination in repeated surveys much easier. For this reason, Poisson sampling is increasingly used in business surveys conducted by statistical agencies. For example, Poisson sampling is going to be used in the redesign of about 100 Statistics Canada business surveys. Another reason justifying the use of Poisson sampling is that it allows us to compare two methods for determining the tuning constant: (i) the proposed method, which is based on minimizing (24); and (ii) the usual method that consists of determining the tuning constant that minimizes the estimated mean square error. For the strategy consisting of Poisson sampling and the Horvitz-Thompson estimator, it is easy to obtain an expression for the estimated mean square error without relying on some simplifications that would make the comparison of the two methods difficult.

In each sample, we first computed three Horvitz-Thompson type estimators: (i) the Horvitz-Thompson estimator given by $\hat{\theta}^{\mathrm{HT}}=\sum_{i \in S} d_{i} y_{i}$; (ii) the robust estimator given by 26 , which we denote by $\hat{\theta}_{\mathrm{cb}}^{\mathrm{RHT}}$; (iii) the robust estimator 16 , where the tuning constant $c$ was chosen so that its estimated mean square error was minimized. We denote the resulting estimator by $\hat{\theta}_{\mathrm{mse}}^{\mathrm{RHT}}$. From 28 , the estimated mean square error of $\hat{\theta}^{\mathrm{RHT}}$ in 16 under Poisson sampling is

$$
\begin{aligned}
\operatorname{mse}_{P}\left(\hat{\theta}^{\mathrm{RHT}}\right)= & \sum_{i \in S}\left(1-\pi_{i}\right)\left[y_{i}+\psi\left\{\left(d_{i}-1\right) y_{i}\right\}\right]^{2} \\
& +\max \left(0,\left(\hat{\theta}^{\mathrm{RHT}}-\hat{\theta}^{\mathrm{HT}}\right)^{2}-\sum_{i \in S}\left(1-\pi_{i}\right)\left[\psi\left\{\left(d_{i}-1\right) y_{i}\right\}-\left(d_{i}-1\right) y_{i}\right]^{2}\right) .
\end{aligned}
$$

In addition, we investigated the robustness of the ratio estimator. In this case, estimating the conditional bias requires estimating $\gamma$. We were interested in comparing different estimators of $\gamma$. Thus we computed three ratio type estimators: (i) the standard ratio estimator, $\hat{\theta}^{\mathrm{G}}$, which is a special case of the generalized regression estimator (29) with scalar $x_{i}$ and $\nu_{i}=x_{i}$. Under the above sampling design, the standard ratio estimator can be expressed as $\hat{\theta}^{\mathrm{G}}=\left(\tilde{n} / n_{s}\right) \hat{\theta}^{\mathrm{HT}}$, where $n_{s}$ denotes the observed sample size. (ii) The robust estimator (34), where the conditional bias (32) was estimated in a nonrobust fashion. That is, the unknown regression 
coefficient $\gamma$ was replaced by the weighted least square estimator $\hat{\gamma}$ given by (30). We denote the resulting robust estimator by $\hat{\theta}_{\mathrm{nr}}^{\mathrm{RG}}$. (iii) The robust estimator (34), where the conditional bias (32) was estimated in a robust fashion. The unknown regression coefficient $\gamma$ was replaced by an $M$-estimator using the Huber function as a $\psi$-function with $K=1.345$ as the tuning constant. This constant is different from the tuning constant $c$ that minimizes (24). In the infinite population set-up, the value 1.345 is often used because, asymptotically, the robust estimator of $\gamma$ based on this threshold is $95 \%$ as efficient as the nonrobust estimator if the true distribution is normal. We denote the resulting robust estimator by $\hat{\theta}_{\mathrm{r}}^{\mathrm{RG}}$. In the case $\tilde{n}=10$, a small number of samples contained fewer than two observations, in which case they were discarded.

For comparisons of estimators, we computed the Monte Carlo percent relative bias

$$
\operatorname{RB}_{\mathrm{MC}}(\hat{\theta})=100 \times \frac{E_{\mathrm{MC}}(\hat{\theta})-\theta}{\theta},
$$

where $E_{\mathrm{MC}}(\hat{\theta})=R^{-1} \sum_{r=1}^{R} \hat{\theta}^{(r)}$ with $\hat{\theta}^{(r)}$ denoting the estimator $\hat{\theta}$ in the $r$-th simulated sample, $r=1, \ldots, R$. We also computed the Monte Carlo relative efficiency, using the nonrobust estimator, $\hat{\theta}^{\mathrm{NR}}$, as the reference

$$
\operatorname{RE}_{\mathrm{MC}}(\hat{\theta})=100 \times \frac{\operatorname{MSE}_{\mathrm{MC}}(\hat{\theta})}{\operatorname{MSE} \mathrm{MC}\left(\hat{\theta}^{\mathrm{NR}}\right)},
$$

where $\operatorname{MSE}_{\mathrm{MC}}(\hat{\theta})=R^{-1} \sum_{r=1}^{R}\left(\hat{\theta}^{(r)}-\theta\right)^{2}$.

Table 6 shows the relative bias and the relative efficiency of three HorvitzThompson type estimators. The relative efficiency was computed with respect to the Horvitz-Thompson estimator. As expected, the Horvitz-Thompson estimator, $\hat{\theta}^{\mathrm{HT}}$ showed a negligible bias in all the scenarios. The robust estimators $\hat{\theta}_{\mathrm{cb}}^{\mathrm{RHT}}$ and $\hat{\theta}_{\mathrm{mse}}^{\mathrm{RHT}}$ were slightly or moderately biased, as expected. The robust estimator $\hat{\theta}_{\mathrm{cb}}^{\mathrm{RHT}}$ showed a smaller bias than $\hat{\theta}_{\mathrm{mse}}^{\mathrm{RHT}}$ in all the scenarios. For the populations that did not contain any outlier, both robust estimators were slightly less efficient than the Horvitz-Thompson estimator with a value of relative efficiency ranging from 100 to 108 . For the populations with outliers, the robust estimators were generally more efficient than the Horvitz-Thompson estimator. The benefits of using a robust estimator over the Horvitz-Thompson estimator were much more apparent when the sample size was smaller. For example, with an expected sample size equal to 20 and expected sampling fraction equal to 0.02 , the value of relative efficiency corresponding to $\hat{\theta}_{\mathrm{cb}}^{\mathrm{RHT}}$ was equal to $65 \%$, whereas it was equal to $58 \%$ for $\hat{\theta}_{\mathrm{mse}}^{\mathrm{RHT}}$. For smaller expected sample sizes such as $\tilde{n}=10$ and $\tilde{n}=20, \hat{\theta}$ mse performed better than $\hat{\theta}_{\mathrm{cb}}^{\mathrm{RHT}}$ in terms of relative efficiency, but seemed slightly less efficient than 
$\hat{\theta}_{\mathrm{cb}}^{\mathrm{RHT}}$ for larger expected sample sizes such as $\tilde{n}=50$ and $\tilde{n}=100$. These results suggest that minimizing (24) can be viewed as a good alternative to minimizing the estimated mean square error, while being much simpler to implement in practice. For a given expected sampling fraction, the relative bias of the robust estimators decreased as the expected sample size increased. Also, the relative efficiency approached 100 as the expected sample size increased. These results are evidence that $\hat{\theta}_{\mathrm{cb}}^{\mathrm{RHT}}$ is design-consistent as discussed in Section 3.4 and suggest that $\hat{\theta}_{\mathrm{mse}}^{\mathrm{RHT}}$ is also design-consistent, although we did not provide a formal proof of consistency for the latter.

We now turn to the ratio type estimators. Table 6 shows the relative bias and the relative efficiency, with respect to the standard ratio estimator, of three ratio type estimators. For the populations with no outlier, the standard ratio estimator and the robust estimators showed almost identical performances in terms of both relative bias and relative efficiency. It is worth noting that both robust estimators were never less efficient than the standard ratio estimator. For the populations containing outliers, the robust estimators showed a moderate bias, as expected. Once again, the robust estimators were significantly more efficient than the standard ratio estimator, especially for $\tilde{n} \leq 50$. Once again, the results suggest that both robust versions of the ratio estimator are design-consistent. Finally, it is worth noting that the method of estimating the conditional bias made little difference in terms of relative bias and relative efficiency, especially for $\tilde{n} \geq 50$. This suggests that estimating the conditional bias in a nonrobust fashion may prove useful in practice since it is easy to implement.

\section{Discussion}

We have shown that the conditional bias can be useful for deriving robust estimators in finite population sampling. Implementation of these estimators can be done by modifying either the $y$-values or the design weights of sample units. For instance, the robust estimator $\hat{\theta}^{\mathrm{RHT}}$ given by $(16)$ can be expressed as $\hat{\theta}^{\mathrm{RHT}}=$ $\sum_{i \in s} d_{i} \tilde{y}_{i}$ with $\tilde{y}_{i}=y_{i}-\alpha_{i} d_{i}^{-1} B_{1 i}^{\mathrm{HT}}$ and $\alpha_{i}=1-\psi\left(B_{1 i}^{\mathrm{HT}}\right) / B_{1 i}^{\mathrm{HT}}$. For Poisson sampling, we have $\tilde{y}_{i}=y_{i}\left\{1-\alpha_{i}\left(1-\pi_{i}\right)\right\}$. Noting that $0 \leq \alpha_{i} \leq 1$ and assuming that $y_{i}>0$ for all $i$, it follows that $y_{i} / d_{i} \leq \tilde{y}_{i} \leq y_{i}$. That is, if the conditional bias for unit $i$ is small, its $y$-value reduces to its original value, $y_{i}$, whereas the value of units with a large conditional bias is reduced. Alternatively, $\hat{\theta}^{\mathrm{RHT}}$ can be written as $\hat{\theta}^{\mathrm{RHT}}=\sum_{i \in s} \tilde{d}_{i} y_{i}$, where $\tilde{d}_{i}=d_{i}-\alpha_{i} y_{i}^{-1} B_{1 i}^{\mathrm{HT}}$. In the case of Poisson sampling, we have $1 \leq \tilde{d}_{i} \leq d_{i}$. Therefore, if the conditional bias for unit $i$ is small, its weight reduces to its original weight, $d_{i}$, whereas the weight of units with a large conditional bias is reduced. The weights $\tilde{d}_{i}$ cannot be smaller than 1 , 


\begin{tabular}{|c|c|c|c|c|}
\hline$f$ & $\tilde{n}$ & $\hat{\theta}^{\mathrm{HT}}$ & $\hat{\theta}_{\mathrm{cb}}^{\mathrm{RHT}}$ & $\hat{\theta}_{\mathrm{mse}}^{\mathrm{RHT}}$ \\
\hline & & \multicolumn{3}{|c|}{ Populations with no outlier } \\
\hline \multirow[t]{3}{*}{0.02} & 10 & $-0.0(100)$ & $-9.9(107)$ & $-11.3(108)$ \\
\hline & 20 & $-0.0(100)$ & $-5.0(104)$ & $-6.0(105)$ \\
\hline & 100 & $0.1(100)$ & $-0.8(100)$ & $-1.3(101)$ \\
\hline \multirow[t]{4}{*}{0.1} & 50 & $-0.0(100)$ & $-1.9(101)$ & $-2.4(102)$ \\
\hline & 100 & $-0.1(100)$ & $-1.0(101)$ & $-1.3(101)$ \\
\hline & 500 & $0.0(100)$ & $-0.2(100)$ & $-0.3(100)$ \\
\hline & & \multicolumn{3}{|c|}{ Populations with outliers } \\
\hline \multirow[t]{3}{*}{0.02} & 10 & $0.4(100)$ & $-15.7(55)$ & $-23.7 \quad(42)$ \\
\hline & 20 & $-0.0(100)$ & $-10.2 \quad(65)$ & $-16.1 \quad(58)$ \\
\hline & 100 & $-0.1(100)$ & $-5.3(93)$ & -10.0 \\
\hline \multirow[t]{3}{*}{0.1} & 50 & $-0.1(100)$ & $-7.5 \quad(72)$ & $-12.3(74)$ \\
\hline & 100 & $-0.0(100)$ & $-4.9 \quad(88)$ & -7.2 (94) \\
\hline & 500 & $-0.0(100)$ & $-1.6(100)$ & $-2.3(103)$ \\
\hline
\end{tabular}

Table 1: Monte Carlo percent relative bias and relative efficiency (in parentheses) of three Horvitz-Thompson type estimators

which is intuitively appealing.

\section{Acknowledgement}

The work of the second author was supported by a grant from the Natural Sciences and Engineering Research Council of Canada. The authors wish to thank the Editor, an Associate Editor and two anonymous referees for their constructive comments.

\section{Appendix 1}

Proof of the existence of a solution to $\Delta(c)=-\left(\hat{B}_{\min }^{\mathbf{H T}}+\hat{B}_{\max }^{\mathbf{H T}}\right) / 2$

Let $b_{(j)}(j=1, \ldots, n)$ be the $j$-th smallest $\hat{B}_{1 i}^{\mathrm{HT}}, i \in S$. Thus, $b_{(1)}=\hat{B}_{\min }^{\mathrm{HT}}$ and $b_{(n)}=\hat{B}_{\max }^{\mathrm{HT}}$. Let also $a_{(j)}, j=1, \ldots, n$, be the $j$-th smallest $\left|\hat{B}_{1 i}^{\mathrm{HT}}\right|, i \in S$. Finally, let $k_{j} \in S$ be the sample unit for which $\left|\hat{B}_{1 k_{j}}^{\mathrm{HT}}\right|$ is the $j$-th smallest $\left(a_{(j)}=\left|\hat{B}_{1 k_{j}}^{\mathrm{HT}}\right|\right)$ and $z_{(j)}$ be the sign of $\hat{B}_{1 k_{j}}^{\mathrm{HT}}\left(z_{(j)} a_{(j)}=\hat{B}_{1 k_{j}}^{\mathrm{HT}}\right)$. It can be observed that, if the Huber 


\begin{tabular}{rrrrrrr}
\multicolumn{1}{c}{$f$} & $\tilde{n}$ & $\hat{\theta}^{\mathrm{G}}$ & $\hat{\theta}_{\mathrm{nr}}^{\mathrm{RG}}$ & $\hat{\theta}_{\mathrm{r}}^{\mathrm{RG}}$ \\
\hline \multirow{6}{*}{0.02} & 10 & $-0.1(100)$ & -0.2 & $(97)$ & $-0.1(100)$ \\
& 20 & $0.0(100)$ & 0.0 & $(97)$ & $0.0(100)$ \\
& 100 & $-0.0(100)$ & -0.0 & $(99)$ & $-0.0(100)$ \\
0.1 & 50 & $0.0(100)$ & -0.0 & $(99)$ & $0.0(100)$ \\
& 100 & $-0.0(100)$ & -0.0 & $(98)$ & $-0.0(100)$ \\
& 500 & $-0.0(100)$ & $0.0(100)$ & $0.0(100)$ \\
\hline \multicolumn{6}{c}{ Populations with outliers } \\
0.02 & 10 & $-0.6(100)$ & -5.8 & $(54)$ & -7.0 & $(48)$ \\
& 20 & $-0.3(100)$ & -4.6 & $(54)$ & -5.1 & $(48)$ \\
0.1 & 100 & $-0.1(100)$ & -4.8 & $(87)$ & -4.6 & $(86)$ \\
& 50 & $0.0(100)$ & -5.1 & $(70)$ & -5.4 & $(68)$ \\
& 100 & $0.1(100)$ & -3.1 & $(82)$ & -3.2 & $(81)$ \\
& 500 & $0.0(100)$ & -1.5 & $(99)$ & -1.4 & $(99)$
\end{tabular}

Table 2: Monte Carlo percent relative bias and relative efficiency (in parentheses) of three ratio type estimators

$\psi$ function is used, the function

$$
\Delta(c)=\sum_{i \in S}\left\{\psi\left(\hat{B}_{1 i}^{\mathrm{HT}} ; c\right)-\hat{B}_{1 i}^{\mathrm{HT}}\right\}=\sum_{j: a_{(j)} \geq c} z_{(j)}\left(c-a_{(j)}\right)
$$

is continuous and piecewise linear in $c(c \geq 0)$. The points where its derivative changes are at $a_{(j)}(j=1, \ldots, n)$. We use this property of $\Delta(c)$ and the intermediate value theorem to show the result. We consider five cases: (i) $b_{(1)}<0$, $b_{(n)}>0$ and $b_{(n)}>-b_{(1)}$ : we have $a_{(n)}=b_{(n)}$ and let $j_{1}$ be the integer such that $a_{\left(j_{1}\right)}=-b_{(1)}$. Thus, $\Delta\left(a_{(n)}\right)=0$ and it is not difficult to show that

$\Delta\left(a_{\left(j_{1}\right)}\right)=-\sum_{j: a_{(j)} \geq a_{\left(j_{1}\right)}}\left(b_{(1)}+a_{(j)}\right) \leq-\left(b_{(1)}+b_{(n)}\right)<-\left(b_{(1)}+b_{(n)}\right) / 2<0=\Delta\left(a_{(n)}\right)$.

By the intermediate value theorem, there exists a solution $c_{\min }$ which is such that $a_{\left(j_{1}\right)}<c_{\min }<a_{(n)}$. (ii) $b_{(1)}<0, b_{(n)}>0$ and $b_{(n)}<-b_{(1)}$ : an argument similar to (i) can be made in this case. (iii) $b_{(1)}<0, b_{(n)}>0$ and $b_{(n)}=-b_{(1)}$ : we can easily see that $c_{\min }$ must be such that $\Delta\left(c_{\min }\right)=-\left(b_{(1)}+b_{(n)}\right) / 2=0$. This equation is satisfied for any $c_{\min } \geq a_{(n)}$. (iv) $b_{(1)} \geq 0$ : we have $\Delta\left(a_{(n)}\right)=0$ and

$$
\Delta(0)=-\sum_{j=1}^{n} b_{(j)} \leq-\left(b_{(1)}+b_{(n)}\right) \leq-\left(b_{(1)}+b_{(n)}\right) / 2 \leq 0=\Delta\left(a_{(n)}\right) .
$$


By the intermediate value theorem, there exists a solution $c_{\min }$ which is such that

$0 \leq c_{\text {min }} \leq a_{(n)}$. (v) $b_{(n)} \leq 0:$ an argument similar to (iv) can be made in this case. Finally, there may be multiple solutions to the equation $\Delta(c)=-\left(b_{(1)}+\right.$ $\left.b_{(n)}\right) / 2$ because $\Delta(c)$ is not necessarily monotone, except for cases (iv) and (v) which yield a unique solution.

\section{References}

BeAumont, J.-F. \& PATAK, Z. (2012). On the generalized bootstrap for sample surveys with special attention to Poisson sampling. Int. Stat. Rev. 80, 127-148.

Beaumont, J.-F. \& Rivest, L.-P. (2009). Dealing with outliers in survey data. In Handbook of Statistics. Vol. 29, Sample Surveys: Theory, Methods and Inference, Ed. D. Pfeffermann \& C.R. Rao, pp. 247-279. Amsterdam: Elsevier BV.

Campbell, C. (1980). A different view of finite population estimation. Proc. of the Section on Survey Research Methods, Am. Stat. Assoc., 319-324.

Chambers, R. R. (1986). Outlier robust finite population estimation. J. Am. Stat. Assoc. 81, 1063-1069.

Demnati, A. \& RAO, J. N. K. (2004). Linearization variance estimators for survey data. Survey Methodol. 30, 17-26.

DEVILLE, J.-C. (1999). Variance estimation for complex statistics and estimators: linearization and residual techniques. Survey Methodol. 25, 193-203.

Gwet, J.-P. \& Rivest, L.-P. (1992). Outlier resistant alternatives to the ratio estimator. J. Am. Stat. Assoc. 87, 1174-1182.

HÁJEK, J. (1981). Sampling from a finite population. New York: Marcel Dekker.

HAMPEL, F. R. (1974). The influence curve and its role in robust estimation. $J$. Am. Stat. Assoc. 69, 383-393.

Hampel, F. R., Ronchetti, E. M., Rousseeuw, P. J. \& Stahel, W. A (1986). Robust Statistics: the Approach Based on Influence Functions. NewYork: Wiley.

Hulliger, B. (1995). Outlier Robust Horvitz-Thompson estimators. Survey Methodol. 21, 79-87. 
KoKIC, P. N. \& BelL, P. A. (1994). Optimal winsorizing cutoffs for a stratified finite population estimator. J. Offic. Statist. 10, 419-435.

Moreno-Rebollo, J. L., Muñoz-Reyez, A. M. \& Muñoz-Pichardo, J. M. (1999). Influence diagnostics in survey sampling: conditional bias. Biometrika 86, 923-928.

Moreno-Rebollo, J. L., Muñoz-Reyez, A. M., Jimenez-Gamero, M. D. \& MuÑOZ-PICHARDO, J. (2002). Influence diagnostics in survey sampling: estimating the conditional bias. Metrika 55, 209-214.

Muñoz-Pichardo, J., Muñoz-Garcia, J., Moreno-Rebollo, J. L. \& PiÑo-MEJIAS, R. (1995). A new approach to influence analysis in linear models. Sankhya, Series A 57, 393-409.

RaO, J. N. K., Wu, J. \& Yue, K. (1992). Some recent work on resampling methods for complex surveys. Survey Methodol. 18, 209-217.

RoYALL, R. M. (1976). The linear least-square prediction approach to two-stage sampling. J. Am. Stat. Assoc. 76, 657-664.

Schlittgen, R. \& Schwabe, R. (2001). An alternative definition of the influence function. Stat. Prob. Lett. 51, 143-153.

Valliant, R., Dorfman, A. H. \& Royall, R. M. (2000). Finite Population Sampling and Inference: a Prediction Approach. New-York: Wiley.

Zaslavsky, A. M., Schenker, N. \& Belin, T. R. (2001). Downweighting influential clusters in surveys: application to the 1990 post enumeration survey. J. Am. Stat. Assoc. 96, 858-869. 